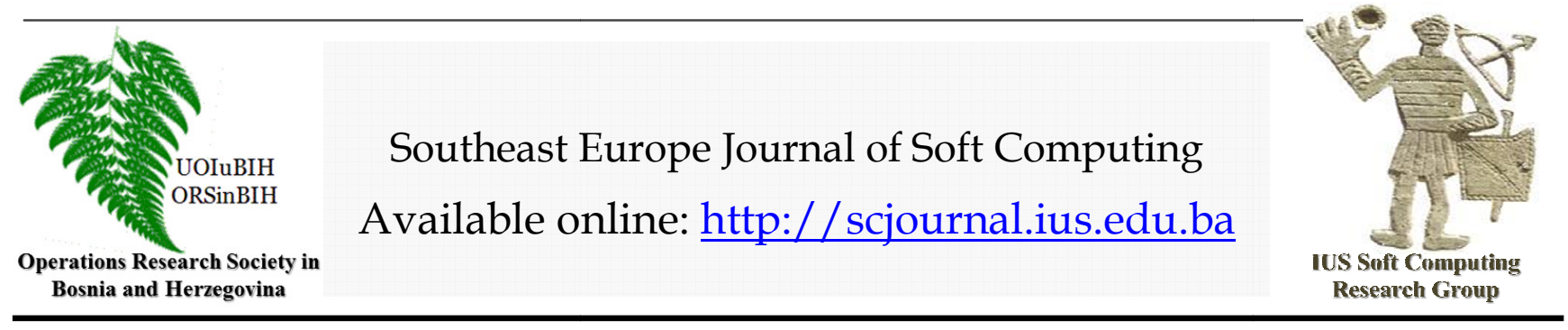

\title{
Hypervariable Regions in 16S rRNA Genes for the Taxonomic Classification
}

\author{
O. Gürsoy \\ M. Can \\ Faculty of Engineering and Natural Sciences, \\ International University of Sarajevo International University of Sarajevo, \\ Hrasnicka Cesta 15, Ilidža 71210 Sarajevo, \\ Bosnia and Herzegovina \\ ogursoy@ius.edu.ba \\ mcan@ius.edu.ba
}

\section{Article Info}

Article history:

Article received on 10 January 2019

Received in revised form 1 February 2019

Keywords:

16S rRNA gene, Variable regions, Taxonomic,

LongestCommonSubsequence

\begin{abstract}
S}$ ribosomal RNA (rRNA) gene sequences are reliable markers for the taxonomic classification of microbes and widely used in environmental microbiology. Production of 16S rRNA gene amplicons in large amounts, encompassing the full length of genes is not yet feasible, because of the limitations of the current sequencing techniques. They are mostly in short reads of length less than 300 base pairs. Hence, the selection of the most efficient hypervariable regions for phylogenetic analysis and taxonomic classification is a current research area. It is found that nine hypervariable regions (V1-V9), resides in bacterial $16 \mathrm{~S}$ ribosomal RNA (rRNA) genes. Family, genus, and species-specific sequences within a given hypervariable region constitute useful targets for diagnostic assays and other scientific investigations. In this study systematic studies that compare the relative advantage of hypervariable regions grouped as V1-V2-V3, V4-V5-V6, and V7-V8-V9 for specific diagnostic goals are done. In the present research, the built in function Longest-CommonSubsequence in computer algebra package MATHEMATICA is used to create an in silico pipeline to evaluate the taxonomic classification sensitivity of the hypervariable regions compared with the corresponding full-length sequences. Conclusions: Our results suggest that V4-V5-V6 region might be an optimal sub-region for the design of universal primers with superior phylogenetic resolution for bacterial phyla.
\end{abstract}

\section{INTRODUCTION}

One of the most important tasks for microbiologists is to profile microbial community to explore bacteria in the environmental niches, and in various ecosystems, since bacteria contribute immensely to global energy conversion and the recycling of matter. To profile microbial community in the samples from human gut is also important since bacteria are an important component of human health.

Unfortunately most bacteria cannot be cultured or isolated under laboratory conditions, and hence our understanding of
Until the development of high-throughput sequencing technology, as mainstream methods in studies of bacterial communities and diversity, terminal restriction fragment length polymorphism (Liu et al., 1997), denaturing gradient gel electrophoresis analysis (Muyzer et al., 1993), fluorescent in situ hybridization (Wagner et al., 1998) and Genechips (He et al., 2012) were used.

Recently, meta-genomic methods just like the use of $16 \mathrm{~S}$ 
sequencing technology (NGST), caused a remarkable expansion of our knowledge regarding uncultured bacteria.

The 16S rRNA gene sequence contains both highly conserved regions for primer design and hypervariable regions to identify phylogenetic characteristics of microorganisms. It is first used in 1985 for phylogenetic analysis (Lane et al., 1985)[9], and marker gene for profiling bacterial communities (Tringe et al., 2008) [10].

Because of the limitation of the sequencing technology, the $16 \mathrm{~S}$ rRNA gene sequences used in most studies are partial sequences, while full-length $16 \mathrm{~S}$ rRNA gene sequences consist of nine hypervariable regions that are separated by nine highly conserved regions (Baker, et al., 2003; Wang, et al., 2009)[11, 12].

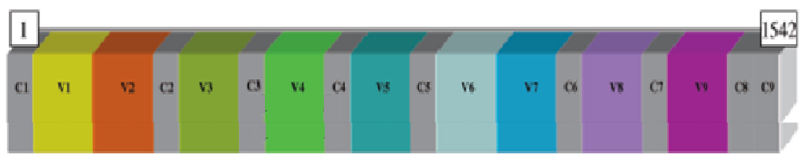

Figure 1. 16S rRNA gene sequences consist of nine hypervariable regions that are separated by nine highly conserved regions.

Positions of the hypervariable sub-regions of the 16S rRNA sequences with their start and end positions as in E. Coli is given in Table 1, as in the below (Yang et al. 2016).

Table 1 Positions of the hypervariable sub-regions of the $16 \mathrm{~S}$ rRNA sequences, their start and end positions (E. Coli)

\begin{tabular}{|l|r|r|r|}
\hline Hyper & \multicolumn{1}{|l|}{ s. position } & \multicolumn{1}{l|}{ e. position } & Length \\
\hline V1 & 8 & 96 & 88 \\
\hline V2 & 97 & 306 & 209 \\
\hline V3 & 307 & 487 & 180 \\
\hline V4 & 488 & 746 & 258 \\
\hline V5 & 747 & 885 & 138 \\
\hline V6 & 886 & 1029 & 143 \\
\hline V7 & 1030 & 1180 & 150 \\
\hline V8 & 1181 & 1372 & 191 \\
\hline V9 & 1373 & 1468 & 95 \\
\hline
\end{tabular}

\section{MATERIALS AND METHODS}

Data contained in the high quality ribosomal RNA databases Greengenes, SILVA, and RDP are downloaded. The number of non-redundant bacterial 16S ribosomal RNA (rRNA) gene sequences with around 1,200 base pairs is 198.510 for Greengenes. This number is 1.488 .662 for SILVA, and 1.350.270 for RDP.

To create a pipeline to annotate unknown bacteria through their 16S rRNA gene short reads, a new similarity between gene sequences is introduced. According to this similarity measure, it is seen that, average in-class similarities are statistically significantly higher than the inter-class averages. It is concluded that this similarity measure can be used to annotate unknown bacteria at all taxon levels.

\subsection{Longest Common Subsequence Search}

To find the level of similarity of two substrings of $16 \mathrm{~S}$ rRNA gene sequences, assume in Figure 1., (a) is a part of a gene reported for a bacteria, and (b) is a part of a gene reported for another, or the same bacteria.

\section{(a) GGCTAACTAGTGTAGAGGTGAAATGATTTAGAT TAGGTGGCAA...}

(b) .GTGTAGAGGTGAAATGCGTAGAT

Figure 2. The longest common subsequence of two genes

The longest common subsequence of (a) and (b) is

\section{GTGTAGAGGTGAAATG}

We note the number of base pairs in this string, then we remove this common subsequence from both sequences. Then look for next longest common substring. If there is no longer one this time the string

\section{TAGAT}

may be the second longest common subsequence. We note also the number of base pairs in this string. This process is repeated till the common substrings are shorter than a threshold. It is seen that ten iterations of this process is optimal.

Then we add the lengths of these common substrings and normalize by dividing this sum, to the length of the shorter string.

\subsection{In-class / Inter-class Similarities}

For the taxonomic classes in family, genus, and species levels, the mean similarity of genes in a class and genes in different classes are computed for 198.510 genes for Greengenes, 1.488.662 genes for SILVA, and 1.350.270 for RDP. The numbers of taxonomic classes in family, genus, and species levels are shown in Table 2. in the below.

Table 2. Numbers of taxonomic classes in family, genus, and species levels.

\begin{tabular}{|l|r|r|r|}
\hline Levels & Greengenes & SILVA & RDP \\
\hline Phylum & 86 & 80 & 51 \\
\hline Class & 232 & 424 & 126 \\
\hline Order & 366 & 843 & 391 \\
\hline Family & 466 & 2117 & 110 \\
\hline Genus & 1949 & 5317 & 354 \\
\hline Species & 2389 & 183284 & \\
\hline
\end{tabular}


Average in-class and inter-class similarities are computed in family, genus, and species levels. The results are shown in Table 3. in the below.

Table 3. Average in-class and inter-class similarities in family, genus, and species levels.

\begin{tabular}{|l|c|r|r|r|r|r|}
\hline & \multicolumn{2}{|c|}{ Family } & \multicolumn{2}{c|}{ Genus } & \multicolumn{2}{c|}{ Species } \\
\hline Databases & In & Between & \multicolumn{1}{c|}{ In } & Between & In & Between \\
\hline Green & 51.34 & 19.34 & 54.48 & 14.94 & 71.69 & 17.46 \\
\hline SILVA & 46.37 & 16.87 & 75.26 & 20.26 & 33.82 & 13.23 \\
\hline RDP & 58.80 & 27.64 & 42.94 & 21.44 & - & - \\
\hline Mean & 50.17 & 21.28 & 57.56 & 18.88 & 52.76 & 15.35 \\
\hline
\end{tabular}

It is seen that the difference between average in-class and inter-class similarities in family, genus, and species levels are statistically significant, and is used in another article to annotate unknown bacteria (Gursoy, and Can, 2019). It is natural to expect that these differences will also be reflected to shorter segments, hypervariable regions.

The nine hypervariable regions are grouped as V1-V2-V3, V4-V5-V6, and V7-V8-V9. Using the sub strings that approximately cover each these groups, the annotation of sampled genes are annotated in three taxonomic levels family, genus, and species.

\section{RESULTS AND DISCUSSION}

When all sub strings that approximately cover each of these three groups are used to annotate sampled genes in three taxonomic levels family, genus, and species a satisfactory accuracy is reached.

Table 4. Average accuracy of annotation of sampled genes by the use of short strings that cover hypervariable regions in the taxonomic level species

\begin{tabular}{|l|r|r|r|r|}
\hline & Whole & VI-V2-V3 & V4-V5-V6 & V7-V8-V9 \\
\hline Greengenes & 73.19 & 69.13 & 66.70 & 61.66 \\
\hline SILVA* & 62.25 & 66.00 & 61.50 & 61.25 \\
\hline
\end{tabular}

$*$ The species for which at least 100 genesreported are cosidered

Table 5. Average accuracy of annotation of sampled genes by the use of short strings that cover hypervariable regions in the taxonomic level genus

\begin{tabular}{|l|r|r|r|r|}
\hline & Whole & VI-V2-V3 & V4-V5-V6 & V7-V8-V9 \\
\hline Greengenes & 78.95 & 87.36 & 86.38 & 77.88 \\
\hline SILVA* & 90.20 & 85.40 & 87.80 & 93.70 \\
\hline RDP & 76.56 & 65.58 & 47.18 & 22.55 \\
\hline
\end{tabular}

*The species for which at least 100 genesreported are cosidered
Table 6. Average accuracy of annotation of sampled genes by the use of short strings that cover hypervariable regions in the taxonomic level family

\begin{tabular}{|l|r|r|r|r|}
\hline & Whole & VI-V2-V3 & V4-V5-V6 & V7-V8-V9 \\
\hline Greengenes & 98.98 & 91.20 & 94.64 & 89.48 \\
\hline SILVA & 74.01 & 67.96 & 77.18 & 69.84 \\
\hline RDP & 64.22 & 73.39 & 66.97 & 29.36 \\
\hline
\end{tabular}

From tables 4, 5, and 6 it is seen that it is possible to annotate short reads around 500 base pairs using the three gene libraries with their 198.510 genes for Greengenes, 1.488.662 genes for SILVA, and 1.350.270 for RDP with an acceptable accuracy.

However if the a short reads are less than 500 base pairs, the nnotation accuracy drops down as in Table 7.

Table 7 Average accuracy of annotation of sampled genes by the use of short strings that cover a part symmetrical from the center of the hypervariable region group V4-V5-V6 in the taxonomic levels of the database Greengenes.

\begin{tabular}{|l|r|r|r|r|r|r|}
\hline Levels & \# levels & \multicolumn{1}{c|}{50} & 150 & 300 & 600 & FULL \\
\hline Phylum & 86 & 59 & 76 & 86 & 87 & 94 \\
\hline Class & 232 & 68 & 77 & 83 & 91 & 90 \\
\hline Order & 366 & 63 & 82 & 87 & 90 & 89 \\
\hline Family & 466 & 45 & 84 & 91 & 94 & 95 \\
\hline Genus & 1949 & 35 & 72 & 81 & 87 & 89 \\
\hline Species & 2389 & 26 & 49 & 52 & 64 & 71 \\
\hline
\end{tabular}

From Table 7 it is seen that for a satisfactoryly accurate annotation using Longest Common Substring similarity measure and the three libraries Greengenes, SILVA, and RDP, short reads must not be much less than 500 base pairs. 
26 O. Gursoy, M. Can / Southeast Europe Journal of Soft Computing Vol.8 No.1 March 2019 (23-26)

\section{REFERENCES}

Baker GC, Smith JJ, Cowan DA. Review and re-analysis of domain-specific 16S primers. J Microbiol Methods. 2003; 55(3): 541-55.

He ZL, Van Nostrand JD, Zhou JZ. Applications of functional gene microarrays for profiling microbial communities. Curr Opin Biotech. 2012; 23(3):460-6

Lane DJ, Pace B, Olsen GJ, Stahl DA, Sogin ML, Pace NR. Rapid determination of $16 \mathrm{~S}$ ribosomal RNA sequences for phylogenetic analyses. Proc Natl Acad Sci U S A. 1985;82 (20): 6955-9.

Liu WT, Marsh TL, Cheng H, Forney LJ. Characterization of microbial diversity by determining terminal restriction fragment length polymorphisms of genes encoding $16 \mathrm{~S}$ rRNA. Appl Environ Microbiol. 1997;63(11):4516-22.

Muyzer G, de Waal EC, Uitterlinden AG. Profiling of complex microbial populations by denaturing gradient gel electrophoresis analysis of polymerase chain reactionamplified genes coding for 16S rRNA. Appl Environ Microbiol. 1993;59(3):695-700.

Gursoy, O., and Can, M. (2019) Taxonomic Classification of Bacteria Using Common Substrings, Southeast Europe Journal of Soft Computing Vol.8 No.1 March, (33-39).

Rinke C, Schwientek P, Sczyrba A, Ivanova NN, Anderson IJ, Cheng J-F, et al. Insights into the phylogeny and coding potential of microbial dark matter. Nature. 2013;499 (7459): 431-7.

Tringe SG, Hugenholtz P. A renaissance for the pioneering 16S rRNA gene. Curr Opin Microbiol. 2008;11(5):442-6.

Wagner M, Noguera DR, Juretschko S, Rath G, Koops HP, Schleifer KH. Combining fluorescent in situ hybridization (FISH) with cultivation and mathematical modeling to study population structure and function of ammonia-oxidizing bacteria in activated sludge. Water Sci Technol. 1998;37(45):441-9

Wang Y, Qian P-Y. Conservative fragments in bacterial 16S rRNA genes and primer design for $16 \mathrm{~S}$ ribosomal DNA amplicons in metagenomic studies. PLoS One. 2009; 4(10): e7401.

Yang, B., Wang, Y., 2 and Qian, P-Y. (2016) Sensitivity and correlation of hypervariable regions in 16S rRNA genes in phylogenetic analysis, BMC Bioinformatics 17:135 